\title{
Teledermatology in Times of COVID-19 Confinement: Comparing Patients' and Physicians' Satisfaction by the Standardized Brest Teledermatology Questionnaire
}

\author{
Joachim W. Fluhr ${ }^{a-c}$ Annie Gueguen ${ }^{b}$ Delphine Legoupil ${ }^{a, b}$ Emilie Brenaut $^{a, b}$ \\ Claire Abasq ${ }^{a, b}$ Helena Araújo ${ }^{a}$ Laurent Misery ${ }^{a, b}$ \\ a Univ Brest, LIEN, Brest, France; ${ }^{b}$ Department of Dermatology, CHRU Brest, Brest, France; ${ }^{c}$ Department of \\ Dermatology, Charité - Universitätsmedizin, Berlin, Germany
}

\section{Keywords}

Teledermatology · Dermatology · Quality assessment questionnaire · COVID-19 · Lockdown · Patient-oriented outcome $\cdot$ Satisfaction

\begin{abstract}
The French government imposed the first COVID-19 pandemic lockdown from March 17 until May 11, 2020. Only emergency cases and teledermatology (TD) were allowed in outpatient settings. A standardized questionnaire was developed to compare the satisfaction level of patients and their treating physicians. Our main question was whether the patients would perceive TD as a valid alternative for direct physical face-to-face consultation. Eighty-two patients and their 4 treating dermatologists from one dermatology department participated in the study (43 females, 39 males) with a mean age of 46.6 years (SD \pm 23.9 ). The reason for TD was a chronic disease in the majority (87.8\%), and mainly as a follow-up (96.3\%). Regarding satisfaction, almost all categories rated around 9 on a $0-10$ verbal analogue scale. The same level of global satisfaction could be seen between the patients and the physicians as well as for the quality of the patient-physician relation and whether all questions could be addressed during the TC. Physicians showed significantly higher scores than patients only for the category of "length" of the consul-
\end{abstract}

karger@karger.com www.karger.com/drm

Karger $\frac{1}{\%}$

GOPEN ACCESS
(C) 2021 The Author(s)

Published by S. Karger AG, Basel

This is an Open Access article licensed under the Creative Commons Attribution-NonCommercial-4.0 International License (CC BY-NC) (http://www.karger.com/Services/OpenAccessLicense), applicable to the online version of the article only. Usage and distribution for commercial purposes requires written permission. tation. Gender, age, as well as distance between the clinic and home of the patient were not influencing factors for satisfaction. Regarding the technical parameters, the evaluation was mostly comparable for patients and physicians, but overall lower than the relational satisfaction parameters, especially for image quality. Patients were significantly more motivated to continue the TD after the lockdown than their treating dermatologists. We see an interest for implementing TD in specialized centers with chronic patients coming from remote places for regular follow-ups. TD cannot replace in-person patient-physician interaction, but was helpful during the lockdown. As a result, TD might become part of dermatology training to prepare for future lockdown situations.

(c) 2021 The Author(s)

Published by S. Karger AG, Basel

\section{Introduction}

Due to the COVID-19 pandemic, the French government imposed a shutdown of the country from March 17 until May 11, 2020. This included a halt of regular clinical outpatient activities. Only emergency cases and teledermatology (TD) were allowed with a reduction of patientphysician contacts by more than $90 \%$ for outpatients in our department. TD was carried out by means of a video system, telephone calls, and E-mail (including pictures). 
So far, studies evaluating TD as a pathway for patientphysician interaction have mainly studied the technical quality of TD, security, and reimbursement aspects [1]. Recently, TD was used in the follow-up of acne patients during the COVID-19 crisis in Italy [2]. A review analyzed the use of TD in melanoma [3]. The use of TD during COVID-19 was discussed in several publications [4-6]. Since the patients' perspective on patient-physician interaction is part of recent research, including telehealth [7-10], we developed the standardized Brest Teledermatology Questionnaire (BTQ). The aim was to evaluate the acceptance of imposed TD in times of COVID-19 lockdown. The French health system allowed reimbursement of TD during the COVID-19 lockdown. Before lockdown, TDs were infrequently used because of legal restrictions.

The BTQ was developed during the time of COVID-19 lockdown when the regular consultations for outpatients were postponed. In order to maintain the possibility for patients to be in touch with our department (dermatology), a phone and videoconference system was established in combination with E-mails. The aim of the study was to better understand interaction during a TD as a tool in times of crises (COVID-19).

The following questions should be answered:

i. How do patients perceive TD as an alternative for direct physical face-to-face consultation?

ii. How could this system be improved?

iii. Is this system acceptable after the lockdown period?

iv. Is patient perception regarding the TD quality in accordance with the perception of the treating physician?

\section{Materials and Methods}

\section{Brest Teledermatology Questionnaire}

It was intended to test the following hypothesis: (a) the global satisfaction differs between patients and physicians, and the evaluation scores of both groups are correlated; (b) global satisfaction differs between age groups; (c) global satisfaction of the patients is influenced by the distance between the clinic and the home of the patients; (d) satisfaction with the length of TD is higher for physicians than for patients.

We developed the standardized BTQ to: (i) assess sociographic data, motivation for the TD, the technical quality, the category of the referring physician (or self-referral), and whether the TD was the first contact with the department or a follow-up; (ii) the central part consisted of 4 items regarding global satisfaction, physicianpatient relationship, duration of the TD, and whether all medical questions were addressed; (iii) finally, we asked the patients whether they would be interested in continuing the TD (English version; see online suppl. material S1; for all online suppl. materials, see www.karger.com/doi/10.1159/000514029. German and French versions are available upon request).
The central part of the BTQ consisted of ordinal scales graded from 0 to 10 as verbal analogue scales (VAS) rating 4 categories: "general satisfaction with the TD," "quality of the patient-physician relation during the TD," "length of the TD," and "have all questions/concerns been addressed during the TD." The technical quality of the TD was evaluated regarding image, sound, and connection quality on an ordinal 0 - to 10 -point scale. The main part of the BTQ was used both for patients and physicians.

The patients were on average contacted $24-72 \mathrm{~h}$ after their TD appointment by a trained nurse of the department of dermatology. The nurse was familiar with dermatological diseases and the organizational setting of the clinic. In order to avoid a possible response bias, the interviews were carried out by a nurse and not by the treating physician. The call was made after the entire teleconsultation was accomplished. If the treating physician had asked directly, it is conceivable that the patients' answer would be more biased by answering in the direction of social desirability. Choosing a nurse does not exclude completely such a bias, but the likelihood is significantly reduced. The interview required approximately $10 \mathrm{~min}$. The treating physician filled in the BTQ within $48 \mathrm{~h}$ after the TD. The patients were offered to replace their scheduled appointment by teleconsultation on a sequential list. There was no physical consultation during the confinement. Not all patients opted for a teleconsultation.

\section{TD Methods}

For TD, the following systems were in place and used: video consultation (with Apizee ${ }^{\circledR}$, Lannion, France), telephone, and the combination of E-mail (pictures) and telephone. The system incurred some technical problems, which are reflected in 3 patient contacts with very negative technical evaluations. The video system required Google Chrome as the technical platform. The regular clinic E-mail system and normal clinic telephone landline were used to connect with either the cell phone or landline of the patient.

\section{Statistical Methods}

The data were analyzed with Prism 6.0 (GraphPad, USA). Normal distribution was tested using the D'Agostino-Pearson test. Since almost all parameters did not show a normal distribution, we carried out pairwise comparisons with a Mann-Whitney test for unpaired samples. In the case of three or more groups to be compared we selected the Kruskal-Wallis test, and in the case of a positive result alpha-adjusted subsequent pairwise comparison was deployed. For testing the distribution in contingency tables, $\chi^{2}$ tests were performed. The significance level was set at $p<0.05$. Values are given as the absolute number of patients or as the percentage of the respective group. Variance was calculated as the standard deviation (SD).

\section{Results}

\section{Sociographic Data}

Eighty-two patients participated in the study (43 females, 39 males) with a mean age of $46.6 \pm 23.9$ years. Female patients had a mean age of $44.5 \pm 26.7$ years, while male patients were slightly older $(49.0 \pm 20.5$ years $)$ without showing significant differences $(p=0.2779)$. We sort- 


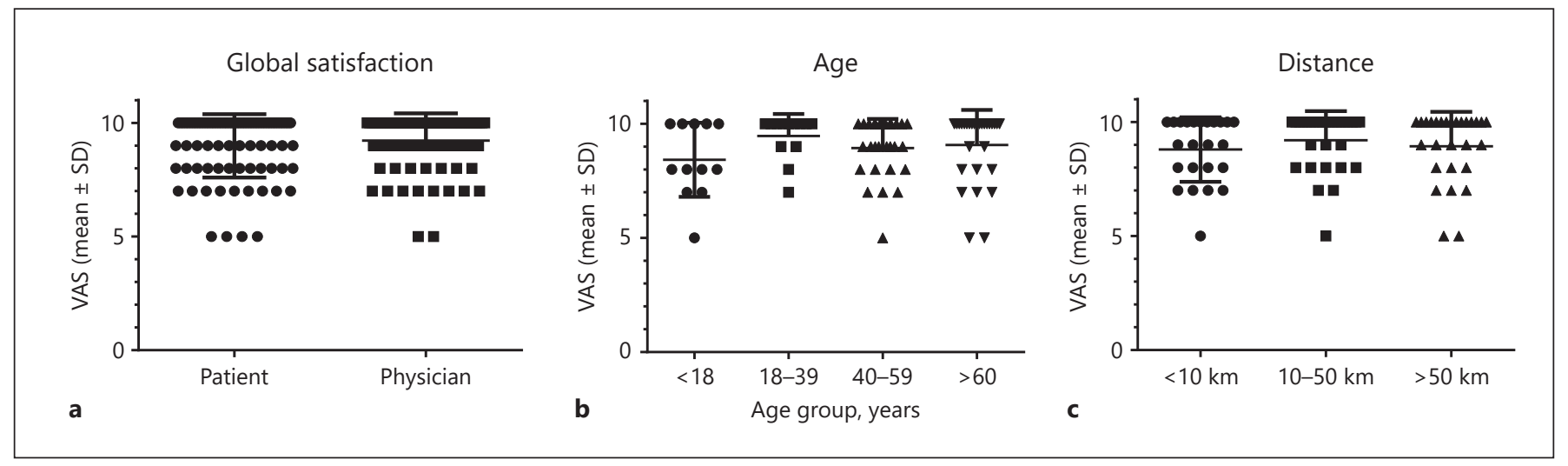

Fig. 1. Global satisfaction scores ( $0-10$ points on a VAS), a The patients' and physicians' global satisfaction score was comparable, with slightly higher satisfaction for the physicians (9.220) compared to the patients (8.988), without reaching statistical significance (Mann-Whitney test, $p=0.3527$ ). The values for patients and physicians correlated significantly (2-tailed Spearman test, $p=0.0331$; patients $n=82$; physicians $n=82)$. $\mathbf{b}$ Global satisfaction clustered by age showed the highest satisfaction score for the 18- to 39 -year age group, but did not reach the level of significance (ANOVA by Kruskal-Wallis test, $p=0.2347$ ). c Global satisfaction clustered by the distance between the clinic and the home of the patients revealed the highest satisfaction scores for the group 10$50 \mathrm{~km}$ away, but did not show significant differences (ANOVA by Kruskal-Wallis test, $p=0.4465)$.

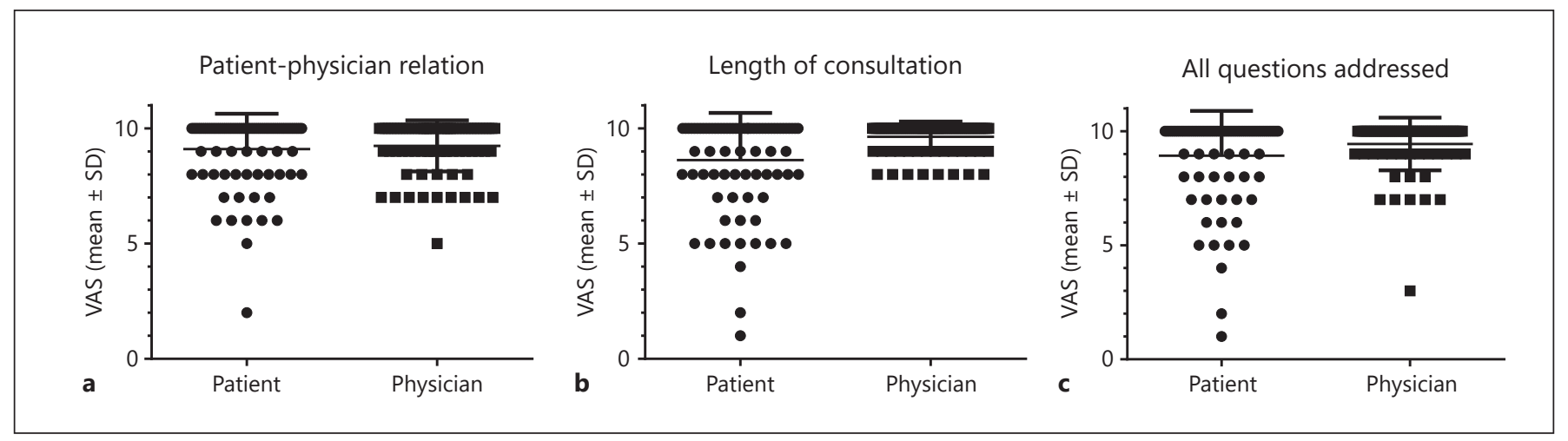

Fig. 2. Quality of interaction parameters (0-10 points on a VAS). a Patient-physician relation score. The physicians showed a slightly but not significantly (Mann-Whitney test, $p=0.7261$ ) higher relation score (9.235) than the patients (9.098; patients $n=82$, physicians $n=81)$. b Length of consultation score. Physicians (9.625) were significantly more satisfied (Mann-Whitney test, $p=0.0021$ ) with the length of the consultation than the patients (8.622) by a full point score (patients $n=82$, physicians $n=80$ ). c All questions addressed score. Physicians reported a higher score in the assessment of whether all questions were addressed (9.438) than the patients (8.927) without reaching statistical significance (MannWhitney test, $p=0.3464$; patients $n=82$, physicians $n=73$ ). ed the population into 4 age groups for further analysis $(<18,18-39,40-59,>60$ years $)$. For underaged patients, the parents answered the interview questions. Female patients had a slightly higher representation in the group aged $<18$ years and an underrepresentation in the group aged $18-39$ years, but did not present significantly different distributions ( $\chi^{2}$ test $p=0.2978$ ).

The travel distance for the patients from their homes to the hospital was divided into 3 categories: $<10 \mathrm{~km}$, which covers the city of Brest and its suburbs $(n=24), 10-50 \mathrm{~km}$, which is the close region within approximately $1 \mathrm{~h}$ of driving $(n=30)$, and $>50 \mathrm{~km}$, corresponding to a larger driving distance of up to $590 \mathrm{~km}$ ( $n=27 ; 1$ answer missing).

\section{Reasons and Referral for Consultation}

The majority reason for a TD was a chronic disease $(n=72 ; 87.8 \%)$. In $7.3 \%(n=6)$ the reason was an acute skin problem, and 4 patients (4.9\%) were seen for unknown diseases. The vast majority of the patients (96.3\%; $n=79$ ) had their TD for follow-up reasons. The referral 


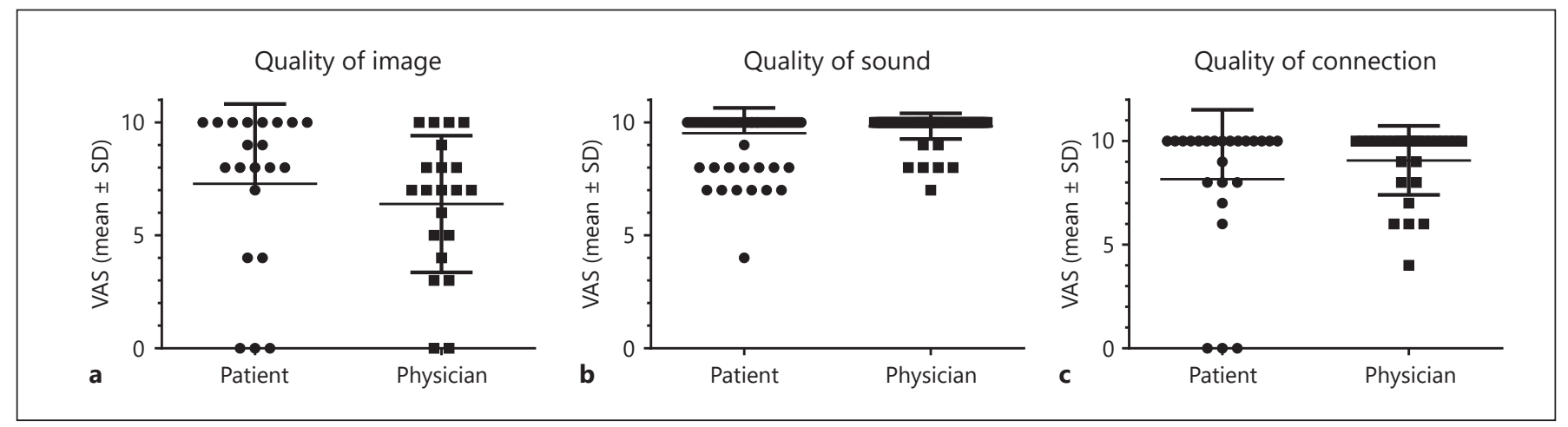

Fig. 3. Technical quality scores ( $0-10$ points on a VAS). a Image quality was scored higher by the patients (7.286) than by physicians (6.381) without reaching statistical significance (MannWhitney test, $p=0.1298$; patients $n=21$, physicians $n=21$ ). $\mathbf{b}$ The sound quality showed higher scores for physicians (9.833) than for patients (9.519), but only as a trend (Mann-Whitney test, $p=$ 0.0635 ; patients $n=81$, physicians $n=78)$. c The connection quality was rated higher by the physicians $(9.069)$ than by the patients (8.167) without reaching statistical significance (Mann-Whitney test, $p=0.5326$; patients $n=24$, physicians $n=29$ ). to our hospital was by general practitioner in $30.5 \%(n=$ $25)$, by dermatologists from private practice in $28.0 \%$ $(n=23)$, by dermatologists from other clinics in $4.9 \%$ $(n=4)$, by other specialists in $14.6 \%(n=12)$, and by selfreferral in $18.3 \%(n=15)$. Three patients gave no indication about their referral source $(3.7 \%)$.

\section{Satisfaction Parameters}

The global satisfaction (Fig. 1) was assessed according to patients versus physicians (Fig. 1a), by age (Fig. 1b), and by distance between the clinic and the home of the patients (Fig. 1c). No significant difference could be detected in these group comparisons. However, the global satisfaction of patients and physicians showed a significant correlation $(p=0.0331)$. All scores were almost at 9 or above 9 in the 0 - to 10 -point scale, indicating a mutual high general satisfaction both in patients and physicians.

Patient-physician relations were reported to be highly satisfactory, with score values above 9 (Fig. 2a) and no significant difference on either side $(p=0.7261)$. The satisfaction with the length of the consultation (Fig. 2b) was rated significantly higher $(p=0.0021)$ by physicians than by patients. The issue of whether "all medical questions were addressed" (Fig. 2c) was rated half a point higher by physicians than by patients, without reaching statistical significance $(p=0.3464)$.

The quality of image, sound, and connection for the TD was evaluated equally for patients and physicians (Fig. 3). Fifty patients used the telephone connection only, 20 used video, and 12 used telephone plus E-mail. Image quality was rated lower by physicians (by almost 1 point), without reaching statistical significance $(p=$
$0.1298)$. No age-related difference in the analysis of the sound was detectable ( $p=0.2928$; data not shown). It has to be noted that not all patients and physicians completed all items in this category since not all used video images. In the image quality category, an overall lower satisfaction score of around 6-7 was noted. No differences between male and female patients were revealed in any of the analyzed parameters (data not shown).

\section{TD in Future Patient-Physician Interaction}

Patients reported significantly more often (44\%) than physicians (13\%) that they would be interested in a future $\mathrm{TD}\left(\chi^{2}\right.$ test $\left.p<0.0001\right)$. Thirty-four answers were missing in the physician group, thus the percentage values for each group were used to calculate the $\chi^{2}$ test.

\section{Discussion}

Sociographic data showed a similar distribution for male and female patients. The age distribution was comparable to the regular population in the outpatient clinic. The vast majority of the patients were already known to the department and were suffering from chronic disease. Approximately a third of the patients came from areas as far as $590 \mathrm{~km}$ away from the clinic. The referrals came from dermatological specialists, general practitioners, other specialties, and self-referrals.

The tested hypothesis regarding the satisfaction parameters of global satisfaction, patient-physician relation, and length of the TD could be answered as follows: (a) the global satisfaction was not different between pa- 
tients and physicians and the evaluation scores of both groups were significantly correlated; (b) the global satisfaction did not differ between different age groups, between patients and physicians, or between male and female patients; (c) there was no impact of the physical distance between the clinic and the home on the global satisfaction of the patients; (d) the satisfaction with the length of the TD was significantly higher for physicians than for patients.

The technical part of the TD showed overall lower satisfaction scores. This is indicative of the need for safe connection modalities that offer a good visual connection and good clinical images to allow for an adequate visual evaluation. Regarding the image quality, the physicians were less satisfied than the patients. The evaluation of the sound quality revealed lower scores for the patients compared to the physicians (as a trend); however, the patient subgroup analysis did not reveal any differences based on age group, which might have been the case for elderly patients with hearing problems.

This is, to our knowledge, one of the first studies evaluating TD regarding the comparative satisfaction of patients and their treating physicians, and more specifically in the context of the COVID-19 lockdown. The collected data indicated a very high satisfaction of both patients and their physicians. In general, both patients and physicians were very satisfied with the interaction-related parameters. The technical quality still needs to be improved. Before the lockdown, TDs were rarely performed in our department because of legal restrictions. We see an indication for TD in specialized centers with chronic patients located in remote places for regular follow-ups. It seems to be important to develop skills that demonstrate patient-centered relationship building [7]. In our opinion TD cannot completely replace direct patient-physician interaction in person.

An online survey among dermatologists analyzed hesitancy, limitations, merits, and the demographic of dermatologists using telemedicine [11]. The authors concluded that while telemedicine in dermatology might not fully replace physical consultation, but it could serve in a supportive role in times of crisis, provided adequate regulatory measures are in place. TD might also become part of dermatology training in order to be prepared for future lockdown situations.

\section{Key Message}

Teledermatology is a valid alternative for direct physical faceto-face consultation, especially in times of pandemic crisis.

\section{Statement of Ethics}

Patients gave oral informed consent to the publication of their anonymized data. The present study is in accordance with the legal framework of the CHRU Brest and as a questionnaire study is exempt from ethical approval.

\section{Conflict of Interest Statement}

J.W.F. - speaker and/or consultant for: Galderma, Sebapharma, Courage \& Khazaka, B. Braun, Expanscience. L.M. - speaker and/or consultant and/or research for: Abbvie, Almirall, Amgen, Bioderma, BMS, Clarins, Clexio, Expanscience, Galderma, Incyte, Janssen, Johnson \& Johnson, Leo, Lilly, Menlo, Novartis, Pfizer, Pierre Fabre, Sanofi, Trevi, UCB. All other authors have no conflicts of interest to declare.

\section{Funding Sources}

This study had no funding. The study was performed as part of the clinical routine obligations.

\section{Author Contributions}

J.W.F. and L.M. designed the study. A.G., D.L., E.B., C.A. and L.M. performed the calls with patients or teledermatology consultations. J.W.F. and H.A. performed the data analysis. All authors contributed to the manuscript.

\section{References}

1 Osman MA, Schick-Makaroff K, Thompson S, Bialy L, Featherstone R, Kurzawa J, et al. Barriers and facilitators for implementation of electronic consultations (eConsult) to enhance access to specialist care: a scoping review. BMJ Glob Health. 2019 Sep; 4(5):e001629.

2 Ruggiero A, Megna M, Annunziata MC, Abategiovanni L, Scalvenzi M, Tajani A, et al.
Teledermatology for acne during COVID-19: high patients' satisfaction in spite of the emergency. J Eur Acad Dermatol Venereol. 2020 Nov;34(11):e662-3.

3 Pala P, Bergler-Czop BS, Gwiżdż JM. Teledermatology: idea, benefits and risks of modern age - a systematic review based on melanoma. Postepy Dermatol Alergol. 2020 Apr;37(2): 159-67.
4 Kumar S, Bishnoi A, Vinay K. Changing paradigms of dermatology practice in developing nations in the shadow of COVID-19: lessons learnt from the pandemic. Dermatol Ther. 2020 Jul;33(4):e13472.

5 Rismiller K, Cartron AM, Trinidad JC. Inpatient teledermatology during the COVID-19 pandemic. J Dermatolog Treat. 2020 Aug; 31(5):441-3. 
6 Bokolo AJ Jr. Use of telemedicine and virtual care for remote treatment in response to COVID-19 pandemic. J Med Syst. 2020 Jun; 44(7):132.

7 Elliott T, Tong I, Sheridan A, Lown BA. Beyond Convenience: Patients' perceptions of physician interactional skills and compassion via telemedicine. Mayo Clin Proc Innov Qual Outcomes. 2020 Jun;4(3):305-14.
8 Baranowski ML, Balakrishnan V, Chen SC. Patient satisfaction with the veteran's administration teledermatology service. J Am Acad Dermatol. 2019 Jan;S0190-9622(19):30143-4.

9 Nicholson P, Macedo C, Fuller C, Thomas L. Patient satisfaction with a new skin cancer teledermatology service. Clin Exp Dermatol. 2020 Aug;45(6):691-8.
10 Wang YC, Ganzorig B, Wu CC, Iqbal U, Khan HA, Hsieh WS, et al. Patient satisfaction with dermatology teleconsultation by using MedX. Comput Methods Programs Biomed. 2018 Dec;167:37-42.

11 Sharma A, Jindal V, Singla P, Goldust M, Mhatre M. Will teledermatology be the silver lining during and after COVID-19? Dermatol Ther. 2020 Jul;33(4):e13643. 